
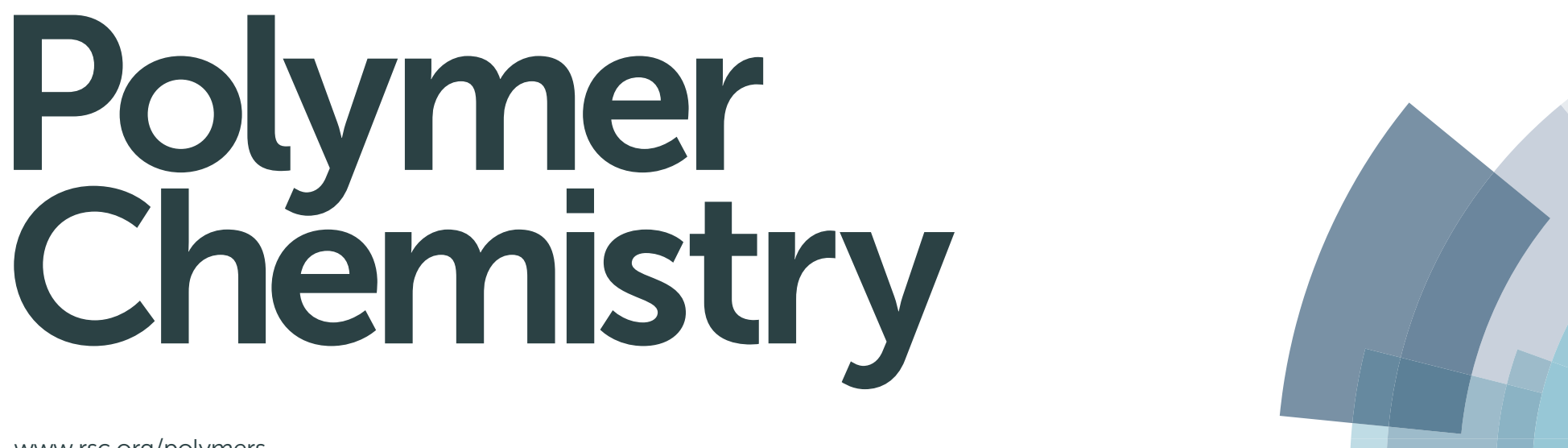

www.rsc.org/polymers

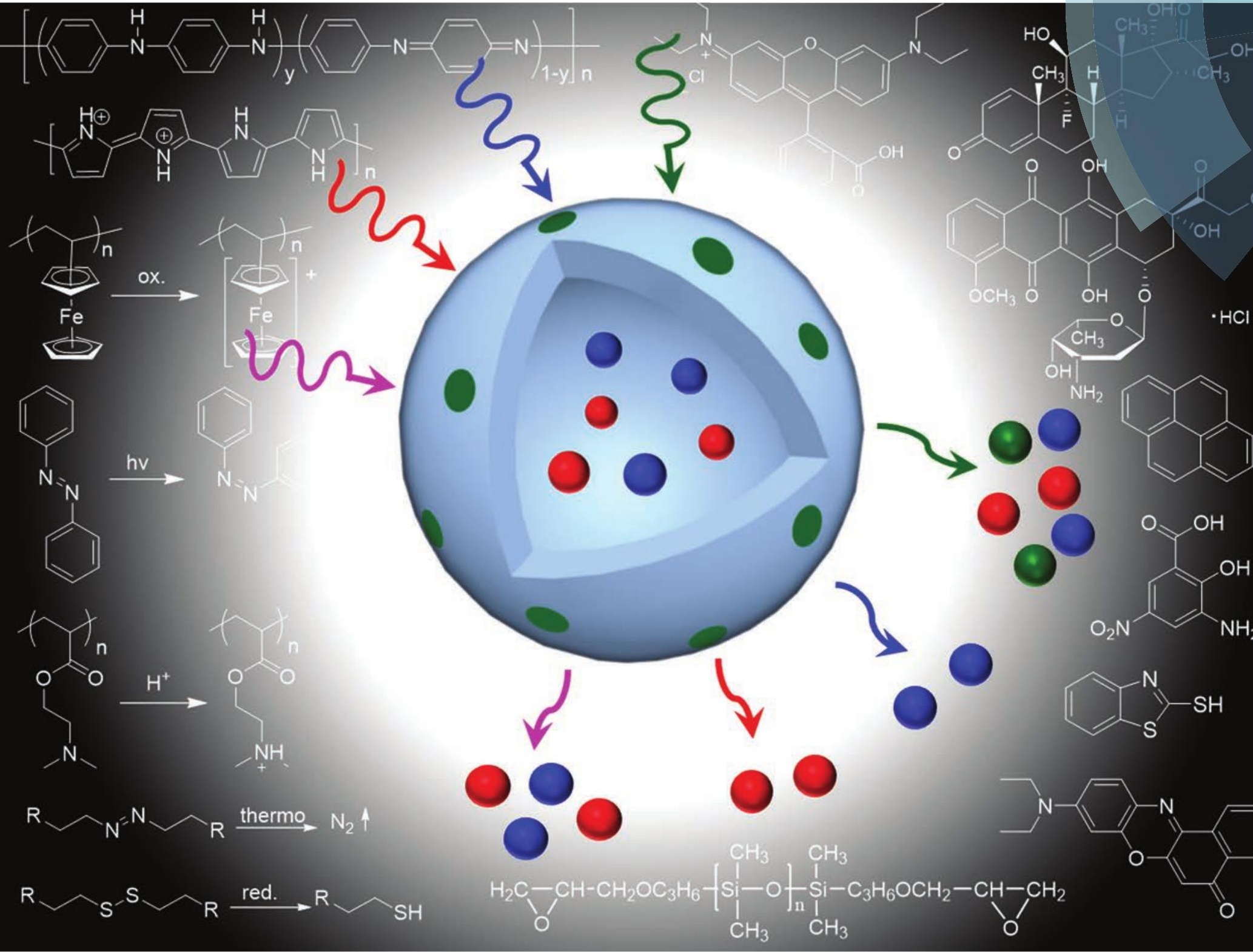

ISSN 1759-9954

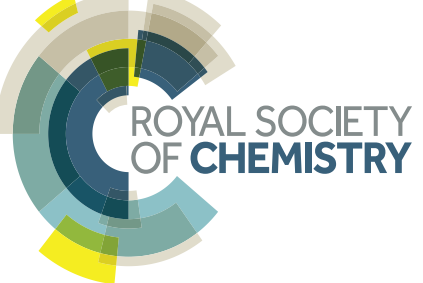




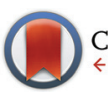

CrossMark \& click for updates

Cite this: Polym. Chem., 2015, 6, 4197

Received 4th March 2015 Accepted 31st March 2015

DOI: $10.1039 /$ c5py00323g

www.rsc.org/polymers

\title{
Advanced stimuli-responsive polymer nanocapsules with enhanced capabilities for payloads delivery
}

\begin{abstract}
Yi Zhao, Li-Ping Lv, Shuai Jiang, Katharina Landfester and Daniel Crespy*
Stimuli-responsive polymer nanocapsules (PNCs) are smart nanocarriers that encapsulate functional payloads and release them on demand upon external triggers. Stimuli-responsive PNCs are of interest in a wide range of disciplines such as pharmaceutics, agriculture, and materials science. Studies on stimuliresponsive PNCs so far are widely reported but are mainly focused on using only one stimulus to release one payload. However, a nanocarrier is efficient if distinct payloads can be selectively released via different stimuli because unwanted and unspecific release can be avoided. Here, the recent progress of stimuli-responsive PNCs that possess enhanced capabilities for payloads delivery is highlighted, including PNCs that respond to multiple stimuli, stimuli-responsive PNCs that co-encapsulate different payloads, and stimuli-responsive PNCs that release the payloads selectively or in a pulsatile way. Finally, the possible future directions in this area are suggested.
\end{abstract}

\section{Introduction}

Polymer nanocapsules (PNCs) are submicron colloidal particles that possess core-shell structures, where the core is an oily or aqueous liquid reservoir and the polymer shell is a protective layer. ${ }^{1,2}$ PNCs can encapsulate large quantities of guest

Max Planck Institute for Polymer Research, Ackermannweg 10, 55128 Mainz, Germany.E-mail: crespy@mpip-mainz.mpg.de

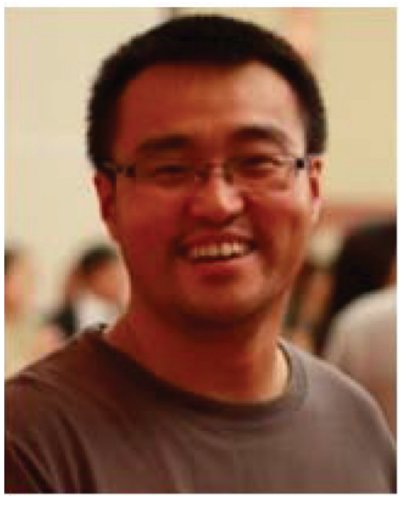

Yi Zhao
Yi Zhao obtained his BEng and MEng degrees in polymer material science from Sichuan University with distinction. In 2010, he earned his PhD degree from University of Sherbrooke, supervised by Prof. Yue Zhao for photo-responsive block copolymers. He did his postdoctoral study in Max Planck Institute for Polymer Research in Mainz with Prof. Katharina Landfester and Dr Daniel Crespy, working on self-healing materials and miniemulsion. In 2013, he joined Institut National de la Recherche Scientifique (EMT centre) in Varennes, working with Prof. Marc A. Gauthier and Prof. Ana Tavares for antibody purification. His current research interests include self-healing materials, polymerbiomacromolecule hybrid materials, and smart drug delivery systems. substances in the core and protect them from undesired leakage. Compared with microcapsules, PNCs have smaller size, higher surface to volume ratio, and usually a higher probability of uptake by cells. Compared with solid nanoparticles, nanocapsules with a liquid core allow for the encapsulation of a much larger amount of payloads. Therefore, PNCs have huge potential applications in drug delivery, ${ }^{3}$ food enhancements, ${ }^{4}$ cosmetics, ${ }^{5}$ actuators, ${ }^{6}$ and self-healing materials. ${ }^{7}$ Stimuliresponsive polymers display sharp property transitions upon slight environmental changes in $\mathrm{pH}^{8},{ }^{8}$ temperature, ${ }^{9}$ electric

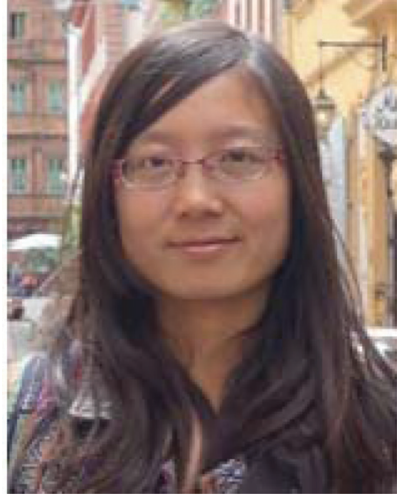

Li-Ping Lv
Li-Ping Lv obtained her BEng and MEng degrees in polymer material science from University of Jinan and Zhejiang University in 2008 and 2011. She completed her PhD in 2014 under the supervision of Prof. K. Landfester and $\mathrm{Dr}$ D. Crespy in the Max Planck Institute for Polymer Research in Mainz. She is now doing her postdoctoral study in the same group. Her current research interests include stimuli-responsive materials for self-healing in anticorrosion and energy storage application. 
field, ${ }^{10}$ redox, ${ }^{11,12}$ light, ${ }^{13}$ or gas concentration ${ }^{14}$ and are therefore used to build PNC shells.

Living polymerizations ${ }^{15-19}$ and post-polymerization modification $^{20,21}$ techniques have largely increased the range of stimuli-responsive polymers, and thus enrich the choices of building blocks for PNCs. The triggered dissociation of PNCs shell is a straightforward route for burst release of payloads from PNCs. Stimuli-responsive polymers with sharp polarity changes $^{22,23}$ upon stimulation or self-immolative polymers ${ }^{24}$ are usually applied to build such types of PNC shells. Selfimmolative polymers are defined as polymers able to react to different environmental stimuli by giving amplified response outputs. $^{25}$

Most of the studies on PNCs are focused on using one trigger to release one payload. However, the practical applications of stimuli-responsive PNCs are usually in complex environments. In some cases, multiple stimuli are requested for efficient release of payloads from PNCs. In other cases, multiple payloads need to be co-encapsulated in PNCs and released together. In addition, pulsatile release of payloads from smart PNCs is particularly interesting in nanomedicine, especially for treating chronic diseases to relieve patients pains caused by frequent injections.

Herein, we discuss the frontiers and breakthroughs in the development of advanced stimuli-responsive PNCs. The release of payloads from polymer capsules triggered by one stimulus has been already well documented in a recent review. ${ }^{26}$ In the present review, we describe stimuli-responsive
PNCs that response to multiple stimuli, encapsulate multiple payloads, and release payloads in pulsatile or selective way.

\section{Encapsulation of payloads in smart PNCs with the miniemulsion process}

Functional payloads can be encapsulated in stimuli-responsive PNCs by different methods including nanoprecipitation, coacervation, emulsion-solvent diffusion or evaporation, inclusion complexation, spray drying, and freeze drying. ${ }^{27,28}$ Layer-bylayer assembly is also feasible for the encapsulation, ${ }^{29}$ but templates and repetitive steps are required. On the contrary, the encapsulation strategies based on miniemulsion (Fig. 1) are

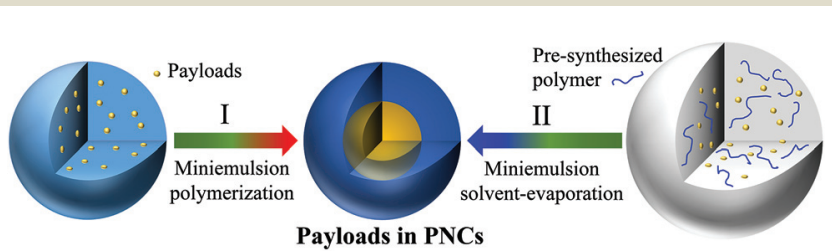

Fig. 1 Schematic illustration for the encapsulation of payloads in smart PNCs based on miniemulsion: (I) miniemulsion droplets containing monomers and non-polymerizable payloads are polymerized to encapsulate payloads in the core; (II) pre-synthesized polymers and payloads are well mixed in a good solvent to form miniemulsion droplets, and then payloads are encapsulated by pre-synthesized polymers after evaporating the good solvent.

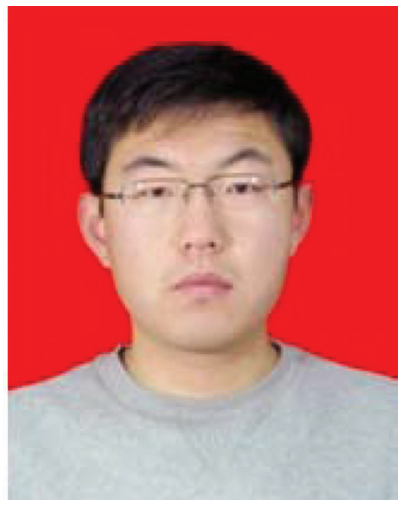

Shuai Jiang
Shuai Jiang studied chemical engineering at the University of Yantai, China. In 2011, he entered the Institute of Coal Chemistry, Chinese Academy of Sciences and researched on new carbon materials/polymer composite materials. In 2013, through the CAS-MPG Doctoral Promotion Programme (DPP), he entered the Max Planck Institute for Polymer Research and is currently pursuing his $P h D$ under the supervision of Prof. Katharina Landfester. His present research concentrates on the synthesis of multicompartment nanofibers through colloid-electrospinning technique and their application in the field of self-healing, anticorrosion, and drug delivery.

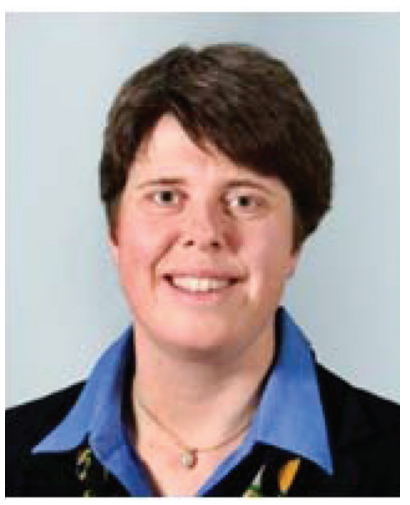

Katharina Landfester
Katharina Landfester studied chemistry at the Technical University of Darmstadt. In 1995, she received her doctoral degree in physical chemistry from the Johannes Gutenberg University of Mainz after working with Prof. H.W. Spiess at the Max Planck Institute for Polymer Research on the synthesis and characterization of core-shell latexes by transmission electron microscopy and solid state NMR. After spending another year as a group leader at the institute, she moved for a doctoral stay at the Lehigh University (Prof. M. El-Aasser) where she first came in contact with the miniemulsion technique. She returned to Germany in 1998 joining the group of Prof. M. Antonietti at the Max Planck Institute of Colloids and Interfaces in Golm. There, she led the miniemulsion group working on new possibilities in the synthesis of complex nanoparticles. In 2002, she got her habilitation in physical chemistry at the University of Potsdam. In 2003, she accepted a chair of full professor of Macromolecular Chemistry at the University of Ulm. Since 2008, she is director at the Max Planck Institute for Polymer Research. 


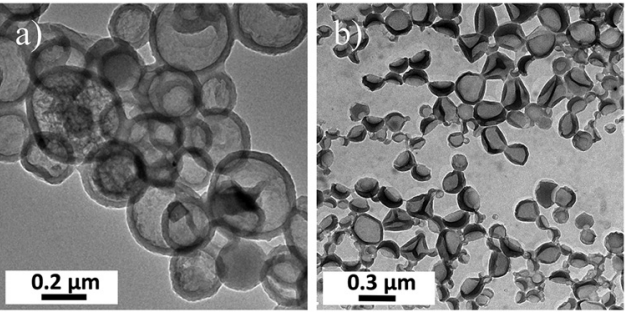

Fig. 2 TEM micrographs of exemplary shown stimuli-responsive PNCs: (a) redox/electrochemically responsive polyaniline nanocapsules prepared by oxidative polymerization of aniline in miniemulsion; (b) photocrosslinkable poly(vinyl cinnamate) nanocapsules prepared by miniemulsion-solvent evaporation technique (adapted from ref. 12 and ref. 7 respectively). Copyright 2013, American Chemical Society and Copyright (2012) Wiley-VCH Verlag GmbH \& Co. KGaA, respectively.

straightforward. The driving force for successful encapsulation is the ideal phase separation between the stimuli-responsive polymer and the payload or the solution containing the payload in miniemulsion droplets, no matter induced by polymerization, ${ }^{30}$ a condensation reaction, ${ }^{31}$ or by solvent evaporation..$^{32}$ In the three different approaches, the formation of the PNCs and the encapsulation of payload are realized simultaneously. Meanwhile, the PNC quantity is easy to scale up by increasing the feed. All approaches can be carried out in direct miniemulsion (usually water as continuous hydrophilic phase) or inverse miniemulsion (usually water as dispersed hydrophilic phase). The encapsulation process benefits from the stability of miniemulsion droplets that are stabilized against Ostwald ripening and coalescence. ${ }^{33}$ For a successful encapsulation of payloads, the interfacial tensions among polymer, payload or its solution, and continuous phase have to be balanced so that the thermodynamically favourable mor-

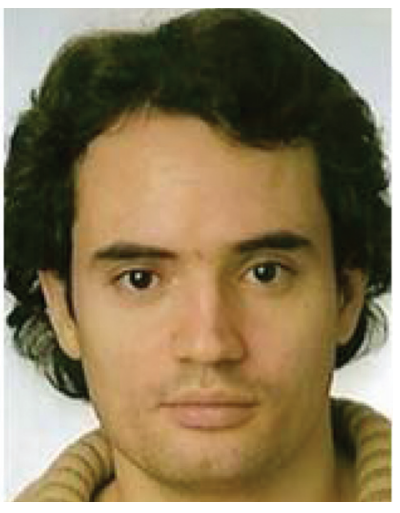

Daniel Crespy
Daniel Crespy studied chemistry at the University of Groningen and Strasbourg where he first got in contact with the field of heterophase polymerization. He joined Professor Katharina Landfester to complete a PhD in the University of Ulm where he developed novel methods to prepare nanocapsules in miniemulsion. In 2006, he held a position as project leader at Empa (Swiss Federal laboratories for Materials Research and Technology), working on stimuli-responsive materials for textile applications. He joined the department of Professor K. Landfester in July 2009 as group leader. His current research interests are the synthesis of new self-healing materials by heterophase polymerizations and the colloid-electrospinning technique. phology to obtain is a core-shell structure as predicted by Torza and Mason. ${ }^{34}$

Miniemulsion polymerization was applied to encapsulate payloads by choosing monomers for constituting units of stimuli-responsive polymers. In direct miniemulsion, hydrophobic payloads are emulsified and the obtained dispersed phase is stabilized by surfactants. The monomers can be either in the dispersed phase, in the continuous phase, or both. After polymerization, the newly formed polymer is soluble neither in the dispersed liquid nor the continuous phase. Thus, it precipitates on the droplet interface to form the PNC shell and encapsulate the payloads at the same time (approach I in Fig. 1). For example, electrochemically responsive polyaniline (PANI) capsules were synthesized by oxidative polymerization of aniline in direct miniemulsion (Fig. 2a); the aniline is mostly soluble in the dispersed phase and precipitates upon polymerization at the interface. ${ }^{12}$ The PANI capsules exhibited redox-responsive properties due to the intrinsic characteristics of the PANI backbone that can be either oxidized or reduced. The encapsulation of different self-healing agents was also achieved. Differently, stimuli-responsive PNCs with primary amine, carboxylic acid and poly(ethylene glycol) as side-chain groups were prepared by free radical miniemulsion polymerization for encapsulating self-healing agents. ${ }^{35}$ Surface initiated polymerization is another feasible approach to attach responsive groups on pre-synthesized PNCs. ${ }^{36}$

The miniemulsion-solvent evaporation technique is another facile method to encapsulate payloads in stimuliresponsive PNCs. ${ }^{32}$ This technique is particularly interesting to fabricate stimuli-responsive PNCs with high purity and low toxicity that can find applications in biomedicine ${ }^{37}$ or for upconversion. ${ }^{38}$ In direct miniemulsion, the pre-synthesized polymer, hydrophobic payload, and a non-solvent for polymer are well mixed in a "good" solvent to form miniemulsion droplets under ultrasonication (approach II in Fig. 1). The volatile low boiling point solvent is immiscible with the continuous phase. ${ }^{39}$ After evaporation of the good solvent, phase separation between the pre-synthesized polymer and the payload in the non-solvent leads to the formation of capsular morphology. Several stimuli-responsive PNCs have been successfully prepared by this approach, including PNCs from biodegradable poly(L-lactide), ${ }^{7}$ photo-crosslinkable poly(vinyl cinnamate) (Fig. 2b), ${ }^{7}$ redox-responsive poly(vinylferrocene)-block-poly(methyl methacrylate) $^{40}$ and $\mathrm{pH}$-/thermo-responsive poly(styrene)-block-poly( $N, N$-dimethylaminoethyl methacrylate). ${ }^{41}$ Principally, this technique can also be used to encapsulate hydrophilic payloads in inverse miniemulsion. ${ }^{42}$

\section{Techniques for monitoring payloads release}

Tracking the payload release profile is a crucial step in the study of smart PNCs. It is beneficial to optimize the design of smart PNCs and selection of stimuli. Before the measurements, the released payloads are usually separated from the 
Table 1 Non-comprehensive list of relevant methods used to monitor the release of payloads from hydrophobic nanocapsules

\begin{tabular}{|c|c|c|c|}
\hline Method & Stimulus & Payload & Ref. \\
\hline \multirow{4}{*}{ Fluorescence } & \multirow{4}{*}{$\begin{array}{l}\text { Ox. } \\
\text { Red. } \\
T, \Delta \text { pH, ox. } \\
\text { Red., ox. }\end{array}$} & Pyrene & 40 \\
\hline & & Nile red & 43 \\
\hline & & Nile red & 41 \\
\hline & & Pyrene, PDMS-DC, PDMS-DE & 12 \\
\hline \multirow[t]{2}{*}{${ }^{1} \mathrm{H}-\mathrm{NMR}$} & $\Delta \mathrm{pH}$ & DDA & 41,44 \\
\hline & Ox. & Diphenylsulfide & 41 \\
\hline \multirow[t]{2}{*}{ FCS } & \multirow{2}{*}{ Red. } & PMI & 45 \\
\hline & & MBT & \\
\hline \multirow[t]{2}{*}{ UV-Vis } & \multirow{2}{*}{$\begin{array}{l}\Delta \mathrm{pH} \\
\text { Red., ox. } \\
\text { Red. }\end{array}$} & 3-NisA & 46 \\
\hline & & MBT & 43 \\
\hline
\end{tabular}

DDA: $N, N$-dimethyldodecylamine, MBT: 2-mercaptobenzothiazole, 3-NisA: 3-nitrosalicylic acid, PDMS-DC: polydimethylsiloxane dicarboxylic acid, PDMS-DE: polydimethylsiloxane diglycidyl ether, PMI: N-(2,6-diisopropylphenyl)perylene-3,4-dicarbonacidimide. Red. = redcution, ox. $=$ oxidation.

PNCs that still contain the unreleased payloads. For example, dialysis bags with suitable molecular weight cut-off value can be used to separate the payloads with low molar mass from the stimuli-responsive PNCs. ${ }^{43}$ The released payloads are then analyzed by several techniques, including high performance liquid chromatography (HPLC), gas chromatography (GC), nuclear magnetic resonance spectroscopy (NMR), ultravioletvisible spectroscopy (UV-Vis), and fluorescence correlation spectroscopy (FCS), to name only a few (see Table 1). Note that many other analytical techniques can be applied to detect the released species. These techniques largely depend on the characteristics of the payloads.

Chromatography, such as HPLC or GC, usually combined with mass spectroscopy, can separate, identify and exactly quantify payloads from a mixture. But this technique is time consuming, costly and generates waste solvent. If there are specific UV-Vis absorption peaks from the payload, UV-Vis spectroscopy can be used to follow the release process. The quantification of released payload can be achieved with the Beer-Lambert equation derived from a calibration curve of the absorbance of pure payload solutions at different concentrations. This technique is fast and inexpensive but it requires that maximum UV-Vis absorption peak of the payloads does not overlap with peaks from other substances such as solvents or side products generated after the trigger of the release. Fluorescence spectroscopy is used to detect the release of fluorescent payload. It can be difficult to quantify the released payload using this technique because the fluorescence of a substance is largely influenced by $\mathrm{pH}$, temperature, solvents and aggregation states of the substance. For payloads having no UV-Vis absorption peaks and fluorescence, ${ }^{1} \mathrm{H}-\mathrm{NMR}$ can be used to track the release process if the released payloads can be separated from the nanocapsules, or if the released payload presents a distinct resonance peak. The latter case was accomplished for example in the case of a hydrophobic tertiary amine that was released by acidifying the medium. ${ }^{41,44}$ Apply- ing an external or internal standard, quantification of released payloads can also be achieved. One general issue during the characterization of the release from nanocapsules is their colloidal stability after being triggered. If they are not stable in dispersion after application of the stimuli, investigation on the release will yield information on the release profile of aggregates rather than release from the nanocapsules. The second classical issue is the separation of released payloads from encapsulated payloads. Since nanocapsules scatter light, it is normally suitable to separate the nanocapsules from their continuous phase after the release of the payloads. However, it has been shown in some cases that centrifugation can be detrimental to the mechanical integrity of the nanocapsules, ${ }^{35}$ thus yielding artificially larger release due to partial destruction of the nanocapsule shell. Dialysis for instance is a mild method allowing for separating released and encapsulated payloads.

\section{Multiple stimuli responsive polymer nanocapsules}

External stimuli provide the driving force to release payloads from PNCs for targeted release. Many studies on stimuliresponsive PNCs deal with only one stimulus such as redox potential, ${ }^{12,40} \mathrm{pH}$ change, ${ }^{46}$ temperature, ${ }^{47}$ or light. ${ }^{48}$ However, several external parameters may change simultaneously in the surrounding medium of the application. For instance, the $\mathrm{pH}$ value and temperature in the human body are variable and both parameters depend on the environment. Thus, $\mathrm{pH}$ value and temperature changes could act simultaneously as stimuli to guarantee the release of drugs from PNCs and to achieve better curative results. The payload release process can be therefore modulated by different parameters. Recent progress in multiple stimuli-responsive PNCs is summarized in Table 2, including the compositions of PNCs, the stimuli types, and sizes of PNCs.

\subsection{PNCs with one sensitive group that respond to multiple stimuli}

Multiple stimuli responsive PNCs can be prepared when the polymer(s) that builds the PNC shell contains sensitive groups that can respond to different triggers. Indeed, there are many homopolymers that can respond to multiple triggers, no matter the sensitive group located in the side-chain or mainchain. For example, poly(2-dimethylaminoethyl methacrylate) (PDMAEMA) with tertiary amine groups in their side-chains can respond to $\mathrm{pH}$, temperature, and $\mathrm{CO}_{2} \cdot{ }^{14}$ Polystyrene- $b$ PDMAEMA nanocapsules were successfully prepared by miniemulsion-solvent evaporation technique. ${ }^{41}$ About $80 \%$ of the encapsulated nile red (NR) was released at $\mathrm{pH} 1.54$ whereas no release of NR was detected at $\mathrm{pH}$ 9.54. Meanwhile, the release of $\mathrm{NR}$ was hindered at neutral $\mathrm{pH}$ value when the temperature was above $75^{\circ} \mathrm{C}$ due to the collapse of the PDMAEMA chains. Multiple stimuli responsive PNCs can also be prepared by using polymers with sensitive groups in the main-chain. For example, polyaniline (PANI) can response to $\mathrm{pH}$, oxidation or 
Table 2 Summary of multiple stimuli responsive polymer nanocapsules

PNC composition

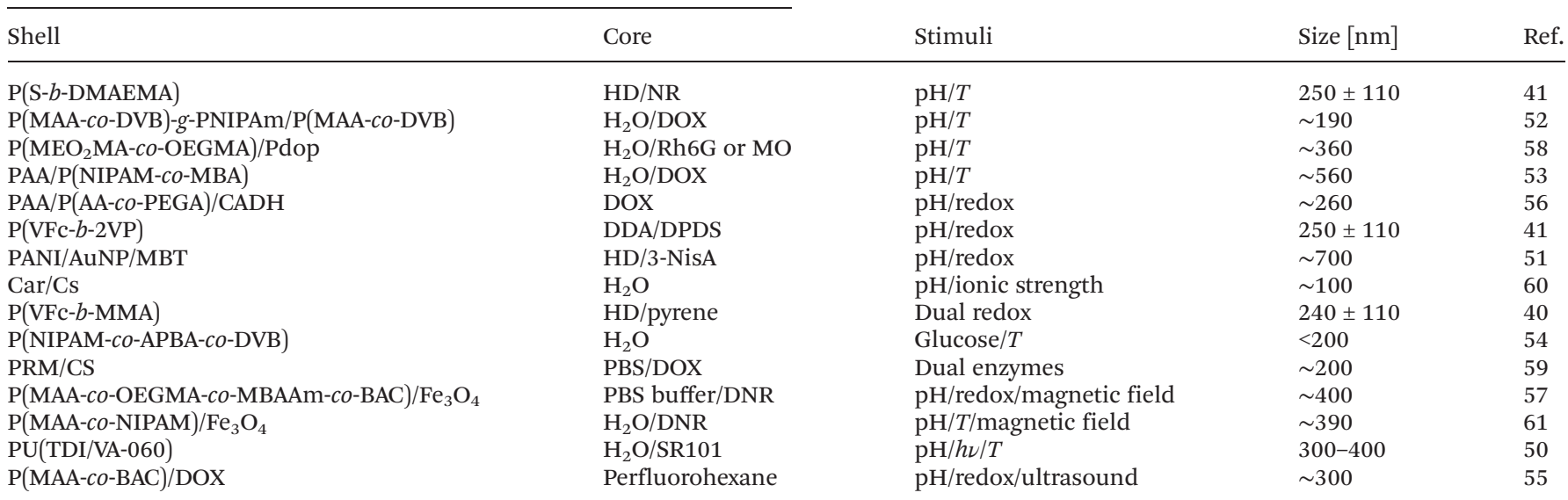

APBA: 3-acrylamidophenylboronic acid; AuNP: gold nanoparticle; BAC: $N, N^{\prime}$-bis(acryloyl)-cystamine; CADH: cystamine dihydrochloride; Car: carrageenan; Cs: chitosan; CS: chondroitin sulphate; DDA: dimethyldodecylamine; DNR: daunorubicin hydrochloride; DOX: doxorubicin hydrochloride; DPDS: diphenyl disulfide; DVB: divinylbenzene; HD: hexadecane; MAA: methacrylic acid; MBAAm: $N, N^{\prime}$-methylene bisacrylamide; MBT: 2-mercaptobenzothiazole; $\mathrm{MEO}_{2} \mathrm{MA}$ : 2-(2-methoxyethoxy)ethyl methacrylate; MMA: methyl methacrylate; MO: methyl orange; NIPAM: $\mathrm{N}$-isopropylacrylamide; 3-NisA: 3-nitrosalicylic acid; NR: nile red; OEGMA: oligo(ethylene glycol) methacrylate; PAA: polyacrylic acid; PANI: poly(aniline); PBS: phosphate buffered saline; PDMAEMA: poly( $N, N$-dimethylaminoethyl methacrylate); PEGA: poly(ethylene glycol) acrylate; Pdop: polydopamine; PRM: protamine; PS: polystyrene; PU: polyurethane; Rh6G: rhodamine 6G; SR101: sulforhodamine 101; TDI: toluene-2,4diisocyanate; VA-060: 2,2'-zzobis\{2-[1-(2-hydroxyethyl)-2-imidazolin-2-yl] propane $\}$ dihydrochloride; VFc: vinylferrocene; 2VP: 2-vinyl pyridine.

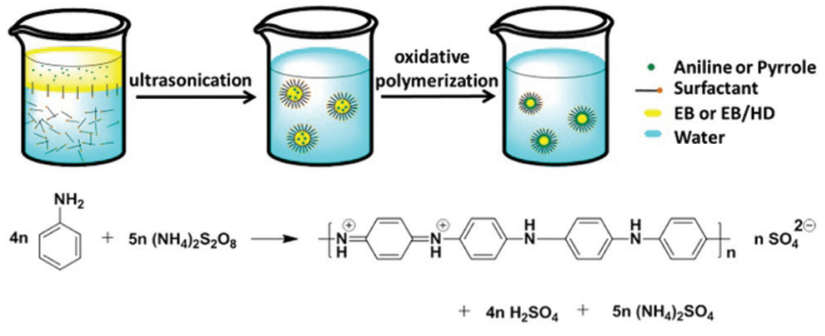

Fig. 3 Scheme illustrating the fabrication of PANI nanocontainers by oxidative polymerization of aniline at the interface of miniemulsion droplets (adapted from ref. 12). Copyright 2013, American Chemical Society.

reduction, and electric field. PANI nanocapsules were prepared by oxidative polymerization of aniline in miniemulsion (see Fig. 3). The encapsulated payloads, diglycidyl ether terminated polydimethylsiloxane (PDMS-DE) and 3-nitrosalicylic acid (3-NisA), were released upon redox $^{12}$ and electrochemical ${ }^{49}$ stimuli, respectively. In the latter case, PANI nanocapsules have been successfully used for self-healing and anticorrosion applications. Payloads in PNCs can be released by the degradation of the polymer main-chain upon triple external stimuli ( $\mathrm{pH}, \mathrm{UV}$ light and temperature). Polyurethane nanocapsules were fabricated by polycondensation between toluene-2,4-diisocyanate (TDI) and a diol containing an azo bond (VA-060) in inverse miniemulsion. ${ }^{50}$ Since the azo unit can respond to $\mathrm{pH}$ change, UV light, and temperature, these stimuli were applied to trigger the release of encapsulated sulforhodamine 101 (SR
101). No release of SR 101 from PNCs was detected upon UV trigger when there were no azo units in the polymer mainchain. The building of multiple stimuli responsive PNCs by using smart homopolymers is conceptually easy to implement. However, there is only a limited number of multiple stimuli responsive homopolymers that can be used to build PNC shell.

\subsection{PNCs with multiple sensitive groups}

Multiple stimuli-responsive PNCs can also be prepared by introducing different sensitive groups in the PNC shell. These sensitive groups can be inserted in the PNCs shell by copolymerization or by directly mixing different stimuli-responsive homopolymers. Random copolymerization of monomers with different sensitive groups is a general and facile method to build smart PNCs.

Such approaches were used to prepare $\mathrm{pH} /$ temperature $^{52,53}$ or glucose/temperature ${ }^{54}$ dual stimuli responsive PNCs. During the copolymerization, the sensitive group can also be placed in a crosslinker. As an example, redox sensitivity can be introduced to multiple stimuli responsive PNCs by using crosslinkers containing disulfide bonds. ${ }^{5-57}$ The removal of the crosslinking by reducing agents induce a burst release of payloads from the PNCs. A special case of PNCs is when the sensitive groups are located in different blocks of a block copolymer. $\mathrm{pH} /$ redox responsive PNCs were prepared by using poly(vinylferrocene)-block-poly(2-vinylpyridine) PVFc- $b$-P2VP. ${ }^{41}$ The encapsulated NR was released fast below $\mathrm{pH} \sim 3$ or in the present of hydrogen peroxide $\left(\mathrm{H}_{2} \mathrm{O}_{2}\right)$ due to the increased permeability of PNC shell. Surface decoration of pre-existing PNCs is another method to build multiple stimuli-responsive PNCs. 
For example, $\mathrm{pH} /$ temperature dual responsive PNCs were prepared by surface initiated atom-transfer radical polymerization of 2-(2-methoxyethoxy)ethyl methacrylate $\left(\mathrm{MEO}_{2} \mathrm{MA}\right)$ and oligo(ethylene glycol) methacrylate (OEGMA) from $\mathrm{pH}$ sensitive poly(dopamine) NCs. ${ }^{58}$ Without further reactions, multiple stimuli responsive PNCs can also be prepared by mixing several stimuli responsive homopolymers to build the PNC shell. Dual enzyme responsive PNCs were built by polypeptide protamine (PRM) and the glycosaminoglycan chondroitin sulphate (CS) by the layer-by-layer technique. ${ }^{59}$ PRM was degraded in the presence of protease enzyme trypsin, while CS was degraded in response to endo- $\beta$ - $N$-acetylhexosaminidase. Thus different enzymes were used to trigger the release of the encapsulated doxorubicin. The aforementioned strategies can be used to build libraries of multiple stimuli responsive PNCs. However, the choices of the building blocks are still limited to polymers.

\subsection{Inorganic/polymer hybrid multiple stimuli responsive nanocapsules}

Inorganic materials have many unique features and advantages which polymer materials do not have. Thus, inorganic/ polymer hybrid nanocapsules have combined functionalities and more tunable parameters for payloads release. For instance, $\mathrm{pH} /$ redox dual responsive PMAA/P(MAA-co-PEGMAco-MBBAm-co-BAC) nanocapsules were prepared by using a sacrificial template-directed approach, and further decorated with magnetic nanoparticles $\left(\mathrm{Fe}_{3} \mathrm{O}_{4}\right) \cdot{ }^{57,61}$ The hybrid nanocapsules displayed efficient magnetic response and could induce hyperthermia and thus the payloads in the nanocapsules could be released by the application of an external magnetic field. Magnetic nanoparticles were also encapsulated in the core or used as building blocks to form a PNC shell for multiple drug delivery. ${ }^{62-64}$ Other inorganic nanoparticles, such as gold nanoparticles (AuNPs) ${ }^{51}$ and quantum dots (QDs), ${ }^{65}$ were also used to build hybrid nanocapsules for anti-corrosion and drug delivery respectively.

\section{Stimuli responsive PNCs with multiple payloads}

The encapsulation of multiple active payloads in PNCs can enrich the functionalities of PNCs. Using multiple payloads together can have also synergistic effect and obtain better applicative results than using the individual components. Thus, loading multiple payloads in PNCs is becoming an emerging research topic in the field of PNCs.

\subsection{Multiple payloads with the same polarity}

Multiple functional payloads with the same polarity, either hydrophilic or hydrophobic, can be directly encapsulated in the PNC core. For example, Staff et al. encapsulated hydrophobic dimethyldodecylamine and diphenyldisulfide in PVFc$b$-P2VP nanocapsules by the miniemulsion-solvent evaporation technique. $^{41}$ Another strategy for loading multiple hydro-

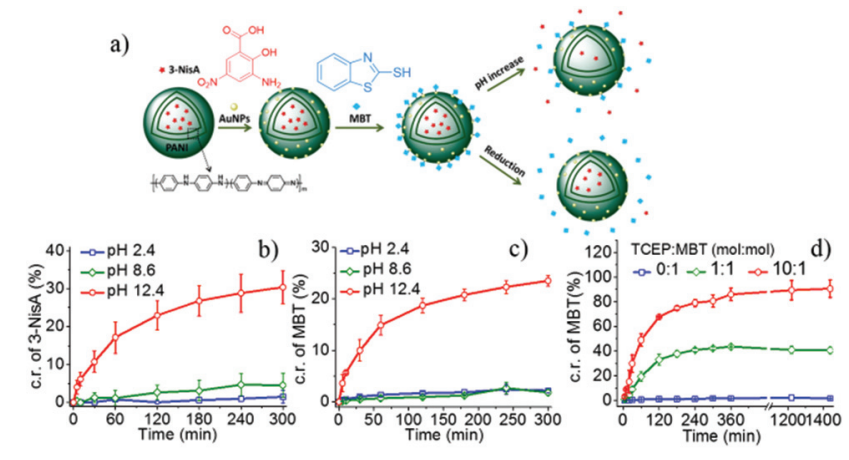

Fig. 4 (a) Scheme illustrating the loading two hydrophobic payloads in PANI nanocapsules and the triggered release of payloads after $\mathrm{pH} /$ chemical reduction stimuli; $(b-d)$ the $\mathrm{pH}$ stimulus was used to trigger the release of MBT and 3-NisA while the reduction was applied to release MBT (adapted from ref. 51). Copyright 2014, American Chemical Society.

phobic payloads is to locate one payload in the core while the other payload is in the shell. As shown in Fig. 4a, 3-NisA was first loaded in the core of PANI nanocapsules during the formation process of the nanocapsules. Then AuNPs were attached on the surface of PNCs via electrostatic interactions, followed by binding 2-mercaptobenzothiazole (MBT) to the AuNPs. ${ }^{51}$ Both payloads were released from PNCs at $\mathrm{pH}$ 12.4, but only MBT was released after treating PNCs by a reducing agent (Fig. 4b-d). MBT and 3-NisA are efficient corrosion inhibitors and can be used for metal anticorrosion. Hydrophobic payloads can also be inserted in the PNC shell through a cleavable weak bond. We recently prepared MBT-containing random and block copolymers, P(MBTMA-co-MMA) and PMBTMA- $b$-PMMA, respectively. The MBT units were attached to the polymer via a disulfide bond. ${ }^{66}$ The polymers were used to encapsulate a hydrophobic self-healing agent, diglycidyl ether polydimethylsiloxane (PDMS-DE), by the miniemulsionsolvent evaporation technique. A chemical reduction was successfully applied to simultaneously release MBT and PDMS-DE from the PNCs. Therefore, these PNCs can be used as selfhealing materials for anticorrosion applications.

\subsection{Multiple payloads with different polarities}

When the payloads are with different polarities, the encapsulation procedure becomes challenging but is still possible. As shown in Fig. 5a, Hu and co-workers successfully encapsulated a hydrophobic and a hydrophilic drug in PNCs with a water-inoil-in-water $(\mathrm{W} / \mathrm{O} / \mathrm{W})$ emulsion strategy. ${ }^{62}$ The PNC shell was composed of poly(vinyl alcohol) (PVA), magnetic nanoparticles $\left(\mathrm{Fe}_{3} \mathrm{O}_{4}\right)$, and a hydrophobic drug paclitaxel (PTX). The core was filled with an aqueous solution of the hydrophilic drug doxorubicin (DOX). Magnetic field was applied to generate local heat through $\mathrm{Fe}_{3} \mathrm{O}_{4}$ nanoparticles, thus leading to fast release of PTX and DOX (Fig. 5c). Similar strategies were employed to encapsulate DOX/PTX or DOX/curcumin that were further modified with trastuzumab ${ }^{63}$ and lactoferrin, ${ }^{64}$ respectively to 
a) $P$
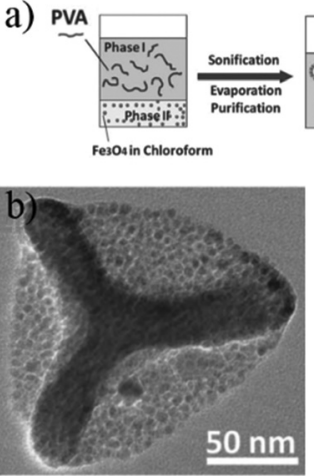
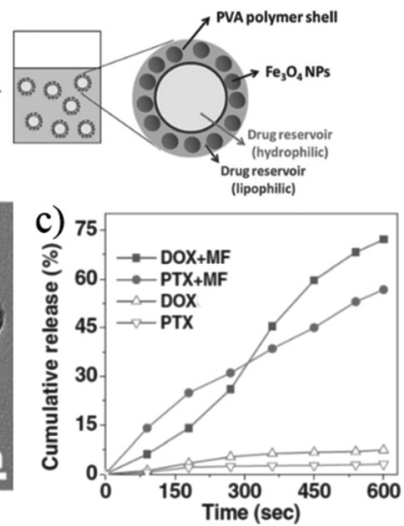

Fig. 5 (a) Scheme showing the encapsulation of a hydrophilic DOX and a hydrophobic PTX in PVA/ $\mathrm{Fe}_{3} \mathrm{O}_{4}$ hybrid nanocapsules; (b) TEM micrograph of the hybrid nanocapsules; (c) plots of the cumulative release of payloads vs. magnetic field (MF) stimulating time (adapted from ref. 62). Copyright (2012) Wiley-VCH Verlag GmbH \& Co. KGaA.

increase cellular uptake of the PNCs. Using a W/O/W emulsion method, hydrophilic dye-labeled DNA or quantum dots (QDs) and hydrophobic pyrene were encapsulated in PAA- $b$-PS/ $/ \mathrm{Fe}_{3} \mathrm{O}_{4}$ hybrid nanocapsules. ${ }^{65}$ High frequency magnetic field was applied to induce pulsatile release of both payloads from PNCs. Besides this approach, template-based method can also be used to load payloads with different polarities. P(MAA-coBAC) nanocapsules were prepared to load hydropholic perfluorohexane in the core and hydrophilic DOX in the shell. ${ }^{55}$ Removal of the disulfide crosslinker by reducing agents could destabilize the structure of PNCs and the two payloads were released.

\section{Precise controlled release with pulsatile and selective delivery}

For smart PNCs, burst release of payloads are widely studied, i.e., payloads are released completely after the trigger. However, it is suitable for some applications to deliver the payloads in a pulsatile way. In other words, the release of payload is suspended after stopping the trigger but it starts again upon reactivation by trigger input. Pulsatile release of drugs from PNCs is especially interesting for the treatment of some chronic diseases. For anticorrosion, a pulsatile release of corrosion inhibitors would ensure that only the needed quantity of payloads is consumed until the corrosion is stopped. The rest of the payload can be then still be used when corrosion occurs again. As mentioned in the previous section, payloads with different polarities were released in a pulsatile fashion under magnetic field trigger. ${ }^{62}$ As another example, Vimalanandan and coworkers encapsulated 3-NisA in PANI nanocapsules and further decorated the PNCs with AuNPs to circumvent Fermi-level misalignment (Fig. 6a). ${ }^{49}$ Electrochemically trigger was applied to release 3-NisA from PNCs in a pulsatile way (Fig. 6b). The cumu-
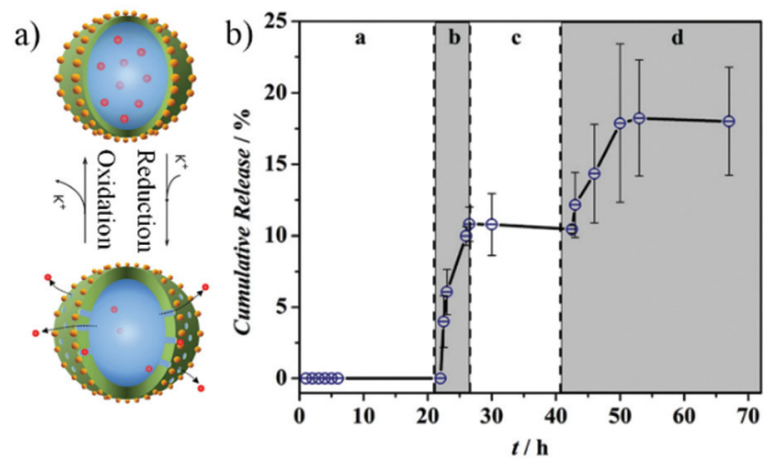

Fig. 6 (a) Scheme illustrating PANI capsule shell that open and close upon external electric stimuli; (b) the cumulative release of 3-NisA was constant when PANI was oxidized (range a and c) whereas the value increased when PANI was reduced (range b and d) (adapted from ref. 49). Copyright (2013) Wiley-VCH Verlag GmbH \& Co. KGaA.

a)
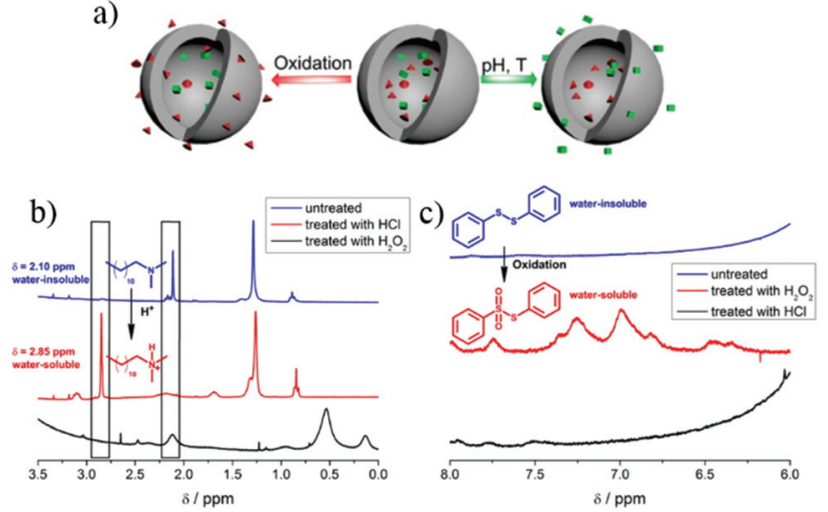

Fig. 7 (a) Scheme illustrating two payloads in PNCs can be selectively released upon different stimuli; (b) ${ }^{1} \mathrm{H}$ NMR spectra showed that DDA was released by treating the PNCs with $\mathrm{HCl}$ and that it was not released in the presence of $\mathrm{H}_{2} \mathrm{O}_{2}$; (c) ${ }^{1} \mathrm{H}$ NMR spectra showed that DPDS was released upon treatment with $\mathrm{H}_{2} \mathrm{O}_{2}$ whereas no release of DPDS was detected in acidic condition (adapted from ref. 41). Copyright 2014, American Chemical Society.

lative release of 3-NisA was constant when PANI was in the oxidized state, but it increased fast when PANI was reduced. Many external stimuli, which can temporarily change the properties of PNC shell, can be applied to achieve pulsatile release of payloads.

The selective release of different payloads in the presence of different triggers is an emerging topic in smart PNCs. As shown in Fig. 4a, the $\mathrm{pH}$ trigger was used to release 3-NisA and MBT together from PNCs, but the reduction trigger was applied to release MBT only. Another representative example is shown in Fig. 7. PVFc- $b$-P2VP nanocapsules were built to encapsulate $N, N^{\prime}$-dimethyldodecylamine (DDA) and diphenyl disulfide (DPDS). DDA was released faster in acidic condition than in oxidative condition (Fig. 7b). In contrast, DPDS was released faster in the present of $\mathrm{H}_{2} \mathrm{O}_{2}$ than that in $\mathrm{HCl}$ 
(Fig. 7c). Thus, the two payloads were selectively released by changing external environments.

\section{Conclusions and outlook}

Substantial progress was achieved for the design of stimuliresponsive PNCs in the recent years. Considering the potential of PNCs in different disciplines, such as medicine, materials science, agriculture, and cosmetics, the study of PNCs will attract more and more attentions in the future. New hybrid PNCs, either inorganic/polymer or biomacromolecule/polymer, will be of special interest because these hybrid PNCs display enhanced modulation parameters, functionalities, or target binding capabilities. The fabrication of multicompartment stimuli-responsive materials from PNCs will allow for the creation of minicomputers, designed for the direct or cascade release of multiple payloads in dependence of the surrounding environments. By implementing stimuli-selective release profiles, they will be truly adaptive materials that can adapt their response to changes in the environment.

\section{Notes and references}

1 P. Couvreur, G. Barratt, E. Fattal, P. Legrand and C. Vauthier, Crit. Rev. Ther. Drug Carrier Syst., 2002, 19, 99-134. 2 X. Huang and B. Voit, Polym. Chem., 2013, 4, 435-443.

3 R. I. El-Gogary, N. Rubio, J. T. W. Wang, W. T. Al-Jamal, M. Bourgognon, H. Kafa, M. Naeem, R. Klippstein, V. Abbate, F. Leroux, S. Bals, G. V. Tendeloo, A. O. Kamel, G. A. S. Awad, N. D. Mortada and K. T. Al-Jamal, ACS Nano, 2014, 8, 1384-1401.

4 M. Chopra, P. Kaur, M. Bernela and R. Thakur, Food Control, 2014, 37, 158-164.

5 J. Hu, Z. Xiao, R. Zhou, Z. Li, M. Wang and S. Ma, Flavour Fragrance J., 2011, 26, 162-173.

6 M. Molberg, D. Crespy, P. Rupper, F. Nuesch, J. A. E. Manson, C. Lowe and D. M. Opris, Adv. Funct. Mater., 2010, 20, 3280-3291.

7 Y. Zhao, J. Fickert, K. Landfester and D. Crespy, Small, 2012, 8, 2954-2958.

8 S. Dai, P. Ravi and K. C. Tam, Soft Matter, 2008, 4, 435-449.

9 R. Liu, M. Fraylich and B. R. Saunders, Colloid Polym. Sci., 2009, 287, 627-643.

10 Q. Yan, J. Y. Yuan, Z. N. Cai, Y. Xin, Y. Kang and Y. W. Yin, J. Am. Chem. Soc., 2010, 132, 9268-9270.

11 Y. Ma, W.-F. Dong, M. A. Hempenius, H. Möhwald and G. J. Vancso, Nat. Mater., 2006, 5, 724-729.

12 L. P. Lv, Y. Zhao, N. Vilbrandt, M. Gallei, A. Vimalanandan, M. Rohwerder, K. Landfester and D. Crespy, J. Am. Chem. Soc., 2013, 135, 14198-14205.

13 Y. Zhao, Macromolecules, 2012, 45, 3647-3657.

14 D. Han, X. Tong, O. Boissiere and Y. Zhao, ACS Macro Lett., 2012, 1, 57-61.
15 J. S. Wang and K. Matyjaszewski, J. Am. Chem. Soc., 1995, 117, 5614-5615.

16 J. Chiefari, Y. K. Chong, F. Ercole, J. Krstina, J. Jeffery, T. P. T. Le, R. T. A. Mayadunne, G. F. Meijs, C. L. Moad, G. Moad, E. Rizzardo and S. H. Thang, Macromolecules, 1998, 31, 5559-5562.

17 F. H. Schacher, P. A. Rupar and I. Manners, Angew. Chem., Int. Ed., 2012, 51, 7898-7921.

18 B. V. K. J. Schmidt, J. Elbert, C. Barner-Kowollik and M. Gallei, Macromol. Rapid Commun., 2014, 35, 708-714.

19 E. Blasco, B. V. K. J. Schmidt, C. Barner-Kowollik, M. Pinol and L. Oriol, Polym. Chem., 2013, 4, 4506-4514.

20 P. Theato, J. Polym. Sci., Part A: Polym. Chem., 2008, 46, 6677-6687.

21 M. A. Gauthier, M. I. Gibson and H. A. Klok, Angew. Chem., Int. Ed., 2009, 48, 48-58.

22 R. H. Utama, M. Drechsler, S. Forster, P. B. Zetterlund and M. H. Stenzel, ACS Macro Lett., 2014, 3, 935-939.

23 F. Cuomo, F. Lopez, A. Ceglie, L. Maiuro, M. G. Miguel and B. Lindman, Soft Matter, 2012, 8, 4415-4420.

24 A. P. Esser-Kahn, N. R. Sottos, S. R. White and J. S. Moore, J. Am. Chem. Soc., 2010, 132, 10266-10268.

25 G. I. Petersson, M. B. Larsen and A. J. Boydston, Macromolecules, 2012, 45, 7317-7328.

26 A. P. Esser-Kahn, S. A. Odom, N. R. Sottos, S. R. White and J. S. Moore, Macromolecules, 2011, 44, 5539-5553.

27 B. V. N. Nagavarma, K. S. Y. Hemant, A. Ayaz, L. S. Vasudha and H. G. Shivakumar, Asian J. Pharm. Clin. Res., 2012, 5, 16-23.

28 P. N. Ezhilarasi, P. Karthik, N. Chanwal and C. Anandharamakrishnan, Food Bioprocess Technol., 2013, 6, 628-647.

29 F. Caruso, R. A. Caruso and H. Mohwald, Science, 1998, 282, 1111-1114.

30 D. Crespy, M. Stark, C. Hoffmann-Richter, U. Ziener and K. Landfester, Macromolecules, 2007, 40, 3122-3135.

31 D. Crespy and K. Landfester, Macromol. Chem. Phys., 2007, 208, 457-466.

32 R. H. Staff, K. Landfester and D. Crespy, Adv. Polym. Sci., 2013, 262, 329-344.

33 D. Schaeffel, R. H. Staff, H. J. Butt, K. Landfester, D. Crespy and K. Koynov, Nano Lett., 2012, 12, 6012-6017.

34 S. Torza and S. G. J. Mason, J. Colloid Interface Sci., 1970, 33, 67-83.

35 J. Fickert, M. Makowski, M. Kappl, K. Landfester and D. Crespy, Macromolecules, 2012, 45, 6324-6332.

36 P. Froimowicz, R. Munoz-Espi, K. Landfester, A. Musyanovych and D. Crespy, Curr. Org. Chem., 2013, 17, 900-912.

37 S. Behzadi, V. Serpooshan, R. Sakhtianchi, B. Muller, K. Landfester, D. Crespy and M. Mahmoudi, Colloids Surf., B, 2014, 123, 143-149.

38 C. Wohnhaas, K. Friedemann, D. Busko, K. Landfester, S. Baluschev, D. Crespy and A. Turshatov, ACS Macro Lett., 2013, 2, 446-450.

39 J. Fickert, C. Wohnhaas, A. Turshatov, K. Landfester and D. Crespy, Macromolecules, 2013, 46, 573-579. 
40 R. H. Staff, M. Gallei, M. Mazurowski, M. Rehahn, R. Berger, K. Landfester and D. Crespy, ACS Nano, 2012, 6, 9042-9049.

41 R. H. Staff, M. Gallei, K. Landfester and D. Crespy, Macromolecules, 2014, 47, 4876-4883.

42 C. Bohlender, K. Landfester, D. Crespy and A. Schiller, Part. Part. Syst. Charact., 2013, 30, 138-142.

43 L. P. Lv, Y. Zhao, K. Landfester and D. Crespy, Polym. Chem., 2015, DOI: 10.1039/C4PY01159G.

44 Y. Zhao, K. Landfester and D. Crespy, Soft Matter, 2012, 8, 11687-11696.

45 J. Fickert, D. Schaeffel, D. Koynov, K. Landfester and D. Crespy, Colloid Polym. Sci., 2014, 292, 251-255.

46 B. Mu, P. Liu and Q. Pu, React. Funct. Polym., 2010, 70, 578584.

47 G. Aguirre, J. Ramos, J. P. A. Heuts and J. Forcada, Polym. Chem., 2014, 5, 4569-4579.

48 Y. Ye, H. T. Xing and Y. Li, Int. J. Nanomedicine, 2014, 9, 4475-4484.

49 A. Vimalanandan, L. P. Lv, T. H. Tran, K. Landfester, D. Crespy and M. Rohwerder, Adv. Mater., 2013, 25, 69806984.

50 E. M. Rosenbauer, M. Wagner, A. Musyanovych and K. Landfester, Macromolecules, 2010, 43, 5083-5093.

51 L. P. Lv, K. Landfester and D. Crespy, Chem. Mater., 2014, 26, 3351-3353.

52 L. Chen, Z. Peng, Z. Zeng, Y. She, J. Wei and Y. Chen, J. Polym. Sci., Part A: Polym. Chem., 2014, 52, 22022216.
53 J. Nan, Y. Chen, R. Li, J. Wang, M. Liu, C. Wang and F. Chu, Nano-Micro Lett., 2014, 6, 200-208.

54 P. Du, B. Mu, Y. Wang and P. Liu, Mater. Lett., 2012, 75, 77-79.

55 P. Yang, D. Li, S. Jin, J. Ding, J. Guo, W. Shi and C. Wang, Biomaterials, 2014, 35, 2079-2088.

56 L. Cui, R. Wang, X. Ji, M. Hu, B. Wang and J. Liu, Mater. Chem. Phys., 2014, 148, 87-95.

57 P. Bilalis, A. Chatzipavlidis, L. A. Tziveleka, N. Boukos and G. Kordas, J. Mater. Chem., 2012, 22, 13451-13454.

58 Z. Ma, X. Jia, J. Hu, G. Zhang, F. Zhou, Z. Liu and H. Wang, Langmuir, 2013, 29, 5631-5637.

59 K. Radhakrishnan, J. Tripathy, D. P. Gnanadhas, D. Chakravortty and A. M. Raichur, RSC Adv., 2014, 4, 45961-45968.

60 Y. Liu, J. Yang, Z. Zhao, J. Li, R. Zhang and F. Yao, J. Colloid Interface Sci., 2012, 379, 130-140.

61 L. A. Tziveleka, P. Bilalis, A. Chatzipavlidis, N. Boukos and G. Kordas, Macromol. Biosci., 2014, 14, 131-141.

62 S. H. Hu, B. J. Liao, C. S. Chiang, P. J. Chen, I. W. Chen and S. Y. Chen, Adv. Mater., 2012, 24, 3627-3632.

63 C. S. Chiang, S. H. Hu, B. J. Liao, Y. C. Chang and S. Y. Chen, Nanomedicine, 2014, 10, 99-107.

64 J. H. Fang, Y. H. Lai, T. L. Chiu, Y. Y. Chen, S. H. Hu and S. Y. Chen, Adv. Healthcare Mater., 2014, 3, 1250-1260.

65 S. H. Hu, S. Y. Chen and X. Gao, ACS Nano, 2012, 6, 25582565.

66 Y. Zhao, R. Berger, K. Landfester and D. Crespy, Small, 2015, DOI: 10.1002/smll.201402521. 